\title{
Muestreo bietápico y diseños muestrales en unidades secundarias evaluados en plantaciones
}

\author{
Two-stage sampling and sampling design in secondary units \\ evaluated in plantations of a joint action forestry program \\ LUIS PATIÑO ${ }^{1}$, VICTOR SANDOVAL ${ }^{2}$ \\ ${ }^{1}$ Programa de Repoblamiento Forestal CORDECO-IC-COTESU, Casilla 975, Cochabamba, Bolivia. \\ ${ }^{2}$ Instituto de Manejo Forestal, Universidad Austral de Chile, Casilla 567, Valdivia, Chile.
}

\section{SUM MARY}

This study evaluates the (a) two-stage sampling method applied (to) in the plantation inventory of the CORDECOIC-COTESU Forestry Program in Cochabamba, Bolivia. The sampling design considers 25 stands of Eucalyptus globulus as primary units. All of them selected with probability proportional to their areas. The secondary units consist of $400 \mathrm{~m}^{2}$ circular plots, randomly chosen, inside each stand. In the second stage, the study also compares the efficiency of four brute volume estimation techniques per unit.

The study concludes that the two-stage sampling method presents limitations in the control of the designed maximum error, even when the selection of primary units and the assignation of secondary units had an adequate adjustment towards the characteristics of joint action forest plantation. The study determines that sampling is the most efficient technique for circular plot levels, with the use of regression estimators.

Key words: two-stage sampling, plantations.

\section{RESUMEN}

Se evalúa el método de muestreo bietápico aplicado en el inventario de plantaciones del programa Forestal CORDECOIC-COTESU en Cochabamba, Bolivia. EI diseño muestral define como unidades primarias 25 rodales de Eucalyptus globulus, los cuales se seleccionan con probabilidad proporcional a su superficie. Las unidades secundarias consisten en parcelas circulares de $400 \mathrm{~m}^{2}$ elegidas aleatoriamente dentro cada rodal muestra. Se compara además la eficiencia de cuatro técnicas de estimación del volumen bruto por unidad en la segunda etapa.

El estudio concluye que el muestreo bietápico muestra limitaciones en el control del error máximo planificado, aun cuando los procedimientos de selección de las unidades primarias y de asignación de las unidades secundarias tienen un ajuste adecuado con las características de las plantaciones de forestería participativa. Al nivel de parcelas circulares se determina que la técnica de mayor eficiencia es el muestreo con uso de estimadores de regresión.

Palabras claves: muestreo bietápico, plantaciones.

\section{INTRODUCCION}

La mayor parte de las plantaciones de forestería participativa establecidas en los valles interandinos y las altiplanicies de Cochabamba, Bolivia, consisten en rodales dispersos y con una amplia variedad de superficies, con predominio de aquellos de extensión inferior a 5 hectáreas.
El inventario de tales poblaciones mediante diseños de muestreo tradicionales y considerando cada rodal como unidad de inventario, se asocia en general con altas intensidades de muestreo ( $\mathrm{Pel} z$ 1980). Por otro lado, si la población definida es un conjunto de rodales, el problema radica en identificar un método válido y práctico para distribuir las unidades muéstrales. 
En este ámbito, otro aspecto limitante de la ejecución de inventarios forestales son los elevados costos de las faenas de terreno. Con relación a los mismos, Bonnor (1972) determina, en el inventario de un área forestal pequeña, que la evaluación de las unidades muéstrales terrestres representa el $32 \%$ del costo total.

Teniendo en cuenta la problemática expuesta, el estudio que se presenta evalúa la compatibilidad del muestreo bietápico con las características de las poblaciones anteriormente descritas y analiza la efectividad de cuatro métodos de estimación del volumen bruto por unidad secundaria. Estos últimos basados en el uso de los árboles como elementos de muestreo.

\section{MATERIAL Y METODO}

Area y población de estudio. Se definió como población de estudio un conjunto de 25 rodales de Eucalyptus globulus $15 \pm 1$ años de edad, con superficies entre 0.2 a 8.3 hectáreas y ubicados desde los 3.280 hasta los $3.630 \mathrm{~m} \mathrm{~s} \mathrm{n} \mathrm{m.} \mathrm{Los}$ mismos se distribuyen en un radio de $5.5 \mathrm{~km}$, pero más del $60 \%$ se encuentran alejados del sistema de accesibilidad. Las plantaciones que suman un total de 57.5 ha pertenecen al núcleo Forestal de Millu Mayu, Provincia Tiraque del Departamento de Cochabamba, Bolivia.

Diseño muestral. La población se sometió a un inventario semidetallado aplicando la técnica del submuestreo o muestreo bietápico. Se estableció como objetivo principal estimar el volumen bruto sólido sin corteza por hectárea $\left(\mathrm{m}^{3} \mathrm{ssc} / \mathrm{ha}\right.$ ) para el rango diamétrico 8 a $26 \mathrm{~cm}$. Las estimaciones quedaron sujetas a un error máximo de $10 \%$ y a un nivel de probabilidad de 0.95 .

$L$ as unidades muéstrales y los métodos utilizados para su selección se detallan a continuación:

- unidades muéstrales

$1^{a}$ etapa rodales de la población

$2^{\text {a }}$ etapa parcelas circulares de $400 \mathrm{~m}^{2}$ (UMS)

- método de selección

$1^{a}$ etapa con reposición y probabilidad proporcional a la superficie de los rodales

$2^{a}$ etapa aleatoria dentro de cada rodal muestra
De acuerdo a Cochran (1978) se efectuó la selección probable de las unidades primarias en ti ocasiones, determinando así un tamaño de submuestra mi, variable en los distintos rodales inventariados. Los parámetros poblacionales se estimaron a través de las ecuaciones del cuadro 1.

\section{CUADRO 1}

Estimadores poblacionales del muestreo bietápico con probabilidad proporcional a la superficie.

Population estímate of the two stage sampling method with probability proportional to surface.

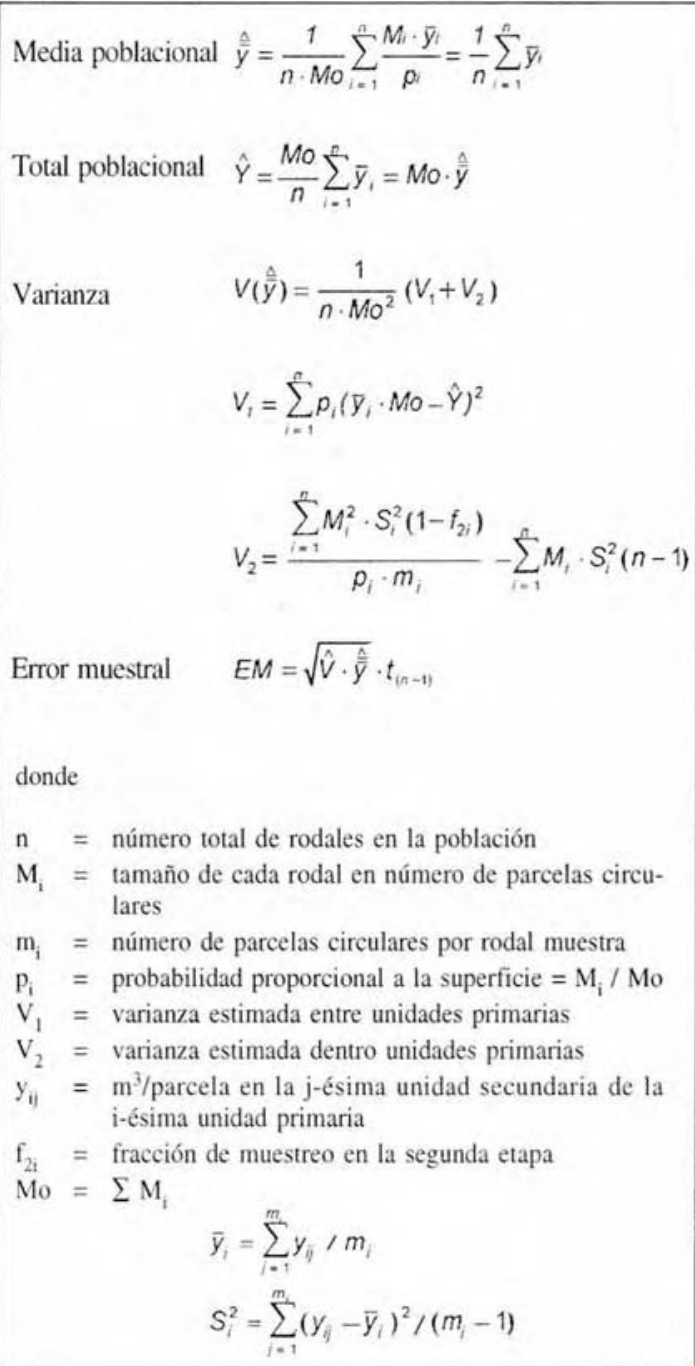

Métodos de estimación del volumen por unidad secundaria. EI volumen total por unidad muestral secundaria (VT/UMS) se estimó mediante los siguientes métodos: 
- muestreo con estimadores de regresión

- muestreo con estimadores de razón

MRg

MRz

- muestreo con probabilidad proporcional a la predicción

M3p

- método tradicional

En todos los casos se consideró como unidades muéstrales los árboles como individuos y se seleccionó a los mismos, a excepción del método $M 3 p$, utilizando un esquema sistemático basado en la proyección de ángulos horizontales desde el centro de la parcela (figura 1).

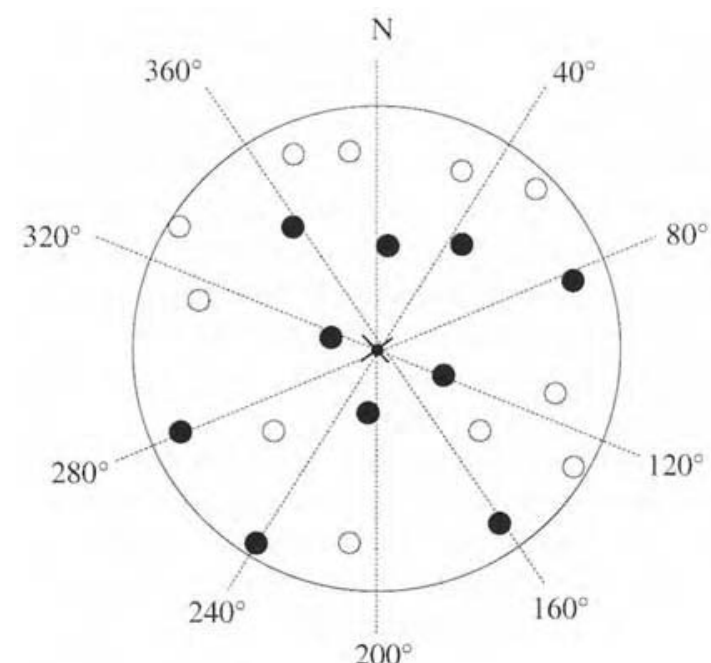

árboles muestra árboles no muestra

- - ángulo horizontal IU $\cdot \mathrm{mi}$ $(\mathrm{i}=1,2,3 \ldots \mathrm{n})$

* centro de parcela circular

) límite de parcela circular

$\mathrm{N}=\mathrm{N}^{\circ}$ total de árboles $/$ parcela $=21$

$\mathrm{n}=\mathrm{N}^{\circ}$ total de árboles muestra/parcela $=10$

$\mathrm{IU}=400^{\circ} / 10=40^{\circ}$

Figura 1. Esquema de procedimiento de selección de árboles muestra.

Process for selecting tree samples.

Los tamaños muestrales quedaron determinados por las técnicas M R g, M R z y M $3 p$ en función del total de árboles en la parcela; en cambio para el método MTr se definió como muestra un núme- ro constante de 10 árboles, para la construcción de una función estimadora de altura total. Adicionalmente, y para los cuatro métodos, se supuso como población aleatoria el conjunto de árboles ubicados dentro de cada parcela.

El método denominado tradicional, el cual no fue catalogado como una técnica de muestreo, comprendió el uso de funciones de altura combinadas con una función general de volumen, con el objeto de estimar primero el volumen por árbol y luego el total por parcela.

Tratándose de las técnicas $M R g$ y $M R z$, el número de árboles muestreados y la estimación de los parámetros por unidad secundaria se efectuó adaptando las ecuaciones presentadas por Scheaffer et al. (1986) y De Vries (1986); en el caso del muestreo $3 p$ se aplicaron los estimadores expuestos por Grosenbaugh (1976). Las estimaciones se rigieron a un error máximo de $10 \%$ y a un nivel de confianza del $95 \%$.

La selección de la técnica muestral de mayor eficiencia se evaluó en base a la magnitud del error muestral (EM \%), la fracción de muestreo (f) y el nivel de costos relacionados (Minutos/ parcelas). Se revisaron además las condiciones exigidas para su aplicación y la incidencia de los reducidos tamaños de muestra utilizados ( $n<30$ árboles) en la fiabilidad de las estimaciones. Elegida la técnica muestral, se la comparó con el método $M T r$ en términos del error absoluto de estimación, de la fracción de muestreo y de los costos respectivos.

\section{RESULTADOS Y DISCUSION}

Método del muestreo bietápico. El procedimiento de selección con reemplazo de las unidades muéstrales de primera etapa y la distribución simultánea de las unidades secundarias permitió en primer término la selección como muestra de sólo 14 rodales de la población. El resultado fue favorable para la reducción de costos de desplazamiento entre estos últimos, dadas su ubicación dispersa y las dificultades de acceso.

EI segundo resultado positivo (figura 2 ), acorde con la teoría de la técnica muestral, fue la selección aproximadamente proporcional de las unidades primarias con respecto a su superficie.

Como lo corroboran las fracciones muéstrales de segunda etapa f2i del cuadro 2. de este modo se 


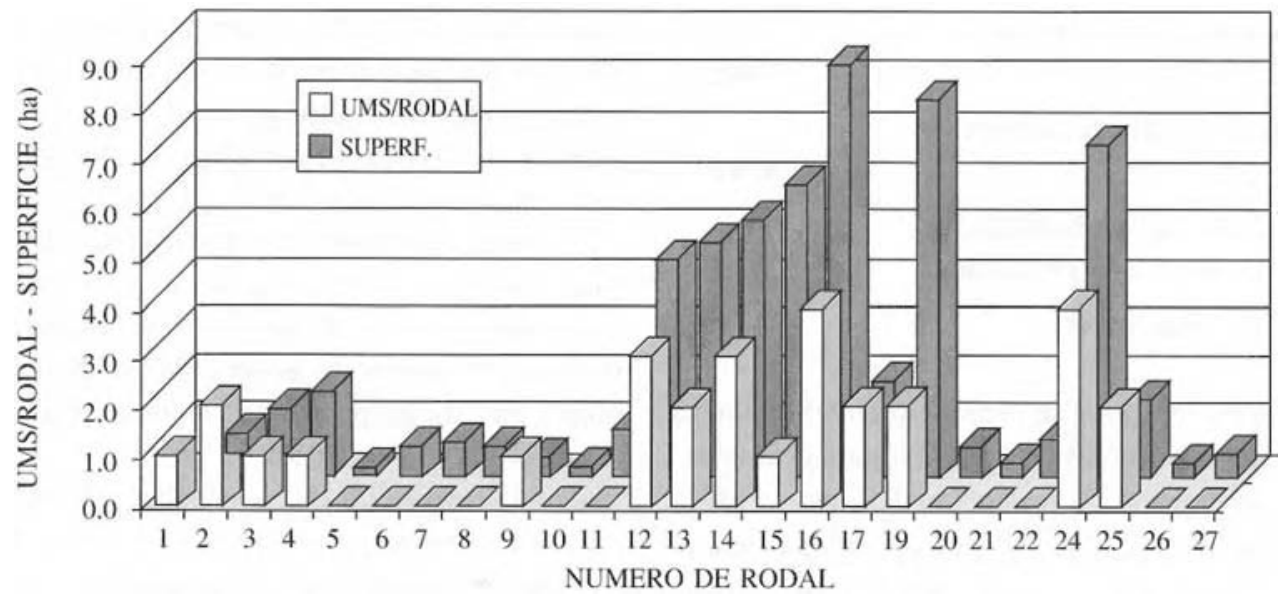

Figura 2: Proporcionalidad de la submuestra mi o UMS/RODAL respecto de la superficie de los rodales. Proportion of the $\mathbf{m i}$ or UMS/Stand sub-sample with regards to surface of the stands.

minimizó el riesgo de una asignación excesiva de parcelas circulares a los rodales más pequeños, y se aseguró un tamaño de submuestra mi o de UMS/ RODAL suficiente para captar la variabilidad de las plantaciones de mayor superficie.

\section{CUADRO 2}

Unidades muestrales secundarias mi y fracción de muestreo f2i por rodal muestra.

mi secondary sampling units and fractions of f2i per stand.

\begin{tabular}{|rrrr|}
\hline $\begin{array}{c}\text { Rodal } \\
\text { muestra }\end{array}$ & $\mathbf{M i}$ & $\mathbf{m i}$ & $\mathbf{f 2 i}$ \\
\hline 01 & 8.2 & $\mathbf{1}$ & 0.12 \\
02 & 22.9 & 2 & 0.09 \\
03 & 34.3 & $\mathbf{1}$ & 0.03 \\
04 & 43.6 & $\mathbf{1}$ & 0.02 \\
09 & 10.8 & $\mathbf{1}$ & 0.09 \\
12 & 111.5 & 3 & 0.03 \\
13 & 120.2 & 2 & 0.02 \\
14 & 132.0 & 3 & 0.02 \\
15 & 148.9 & 1 & 0.01 \\
16 & 207.9 & 4 & 0.02 \\
17 & 48.8 & 2 & 0.04 \\
19 & 190.8 & 2 & 0.01 \\
24 & 168.5 & 4 & 0.02 \\
25 & 39.4 & 2 & 0.05 \\
\hline
\end{tabular}

Métodos de estimación del volumen por unidad secundaria. La revisión de los requisitos de uso de las técnicas muéstrales estableció inicialmente el mejor ajuste de los pares muéstrales de área basal y volumen por árbol a una recta sin trayectoria por el origen, tanto en el muestreo con estimadores de razón como en el muestreo con estimadores de regresión. En ambos casos y en la generalidad de las parcelas las cifras inferiores del error estándar de la estimación $\mathbf{S y x}$ y la superioridad de los coeficientes de determinación $\mathbf{R}^{2}$, que logró la recta sin paso por el origen, fueron los indicativos de la conveniencia de aplicar el muestreo con estimadores de regresión.

Con respecto al método $3 p$, se detectó como problema importante la estimación, en ciertos casos, y la subestimación en otros, del volumen de árbol individual (valores $\mathrm{KPI}$ ), a raíz de la inconsistencia del modelo predictor.

$$
V=0.0006789 \cdot D^{2.6089782}
$$

donde: $V=$ volumen por árbol en $\mathrm{m}^{3} \mathrm{ssc}$ $\mathrm{D}=$ diámetro a $1.3 \mathrm{~m}$

La inconsistencia demostrada por los valores superiores e inferiores a 1 de la razón de corrección Ri se verificó en alrededor del $70 \%$ de las unidades secundarias y se supuso, de acuerdo a Benítez (1992), como fuente de errores significativos en la estimación del volumen total por parcela.

Las hipótesis planteadas y la influencia probable de los tamaños de muestra inferiores a 30 árboles se constataron indirectamente observando el número de ocasiones en las cuales el volumen real por unidad secundaria VTR/UMS quedó capturado por los intervalos de confianza relativos a cada técnica de estimación (cuadro 3). 


\section{CUADRO 3}

Frecuencia de número de parcelas con VTR capturado por intervalos de confianza.

Frequency of plots with VTR according to confidence ranges.

\begin{tabular}{|ccccc|}
\hline \multirow{2}{*}{ M étodo } & \multicolumn{2}{c}{ VTR capturado } & \multicolumn{2}{c|}{ VTR no capturado } \\
\cline { 2 - 5 } & $N^{\circ}$ parc. & $\%$ & $N^{\circ}$ parc. & $\%$ \\
\hline M Rg & 27 & 96.4 & 1 & 3.6 \\
M Rz & 25 & 89.3 & 3 & 10.7 \\
M 3p & 26 & 92.9 & 2 & 7.1 \\
\hline
\end{tabular}

VTR = volumen real por parcela.

La mayor cercanía al $95 \%$ previsto por la teoría de la aproximación normal correspondió a las estimaciones de regresión (método $\mathbf{M R g}$ ), a pesar de que éstas implicaron la superación del error máximo de $10 \%$ en cinco parcelas circulares.

El muestreo con estimadores de regresión se constituyó además en la segunda técnica con menores costos (cuadro 4), como consecuencia directa de la proporción de árboles por unidad secundaria que requirió para generar sus estimaciones (fracción de muestreo f).

También con referencia a los costos de estimación del VT/TJMS, el tiempo excesivo dedicado a la medición de la altura de los árboles muestra MAM justificó la decisión de ejecutar el muestreo dentro de las unidades de segunda etapa.

El ranking ponderado de los métodos, elaborado a partir de los criterios discutidos previamente, señaló como técnica de mayor eficiencia al muestreo con uso de estimadores de regresión, dado su menor puntaje ponderado. En el cuadro 5, relativo a los detalles del ranking, la calificación " 1 " corresponde a la condición más favorable según el criterio; complementariamente se presentan los factores de importancia (ponderación) asignados a estos últimos.

\section{CUADRO 4}

Fracción de muestreo y tiempos/operación promedios por unidad muestral secundaria.

Sampling fractions and time/operation averages per secondary sampling unit.

\begin{tabular}{|c|c|c|c|c|c|c|c|}
\hline \multirow{2}{*}{ Técnica } & \multirow{2}{*}{$f$} & \multicolumn{6}{|c|}{ Tiempos promedios/parcela (min) } \\
\hline & & & $\mathrm{RPC}$ & MTA & UAM & MAM & $\mathrm{TT}$ \\
\hline M Rg & 0.42 & $\%$ & $\begin{array}{r}8.8 \\
17.6\end{array}$ & $\begin{array}{l}13.3 \\
26.6\end{array}$ & $\begin{array}{r}5.4 \\
10.8\end{array}$ & $\begin{array}{l}22.4 \\
44.8\end{array}$ & $\begin{array}{r}50.0 \\
100.0\end{array}$ \\
\hline M Fz & 0.54 & $\%$ & $\begin{array}{r}8.8 \\
14.5\end{array}$ & $\begin{array}{l}13.3 \\
22.0\end{array}$ & $\begin{array}{r}7.1 \\
11.7\end{array}$ & $\begin{array}{l}31.3 \\
51.7\end{array}$ & $\begin{array}{r}60.6 \\
100.0\end{array}$ \\
\hline M 3p & 0.37 & $\%$ & $\begin{array}{r}8.8 \\
18.7\end{array}$ & $\begin{array}{l}13.3 \\
28.2\end{array}$ & $\begin{array}{l}2.1 \\
4.4\end{array}$ & $\begin{array}{l}22.9 \\
48.6\end{array}$ & $\begin{array}{r}47.2 \\
100.0\end{array}$ \\
\hline M Tr & 0.51 & $\%$ & $\begin{array}{r}8.8 \\
15.8\end{array}$ & $\begin{array}{l}13.3 \\
23.9\end{array}$ & $\begin{array}{l}4.1 \\
7.4\end{array}$ & $\begin{array}{l}29.5 \\
53.0\end{array}$ & $\begin{array}{r}55.7 \\
100.0\end{array}$ \\
\hline
\end{tabular}

RPC = replanteo de parcela circular

MTA = medición de DAP de todos los árboles

UAM = ubicación de los árboles muestra

MAM = medición de $\mathrm{H}$ de los árboles muestra

$\mathrm{TT}=$ tiempo total 
LUIS PATIÑO, VICTOR SANDOVAL

\section{CUADRO 5}

Ranking de las técnicas de muestreo en unidades muestrales secundarias.

Ranking of the sampling techniques in secondary sampling units.

\begin{tabular}{|c|c|c|c|c|c|c|}
\hline \multirow{3}{*}{$\begin{array}{c}\text { Criterio } \\
\text { de calificación }\end{array}$} & \multicolumn{5}{|c|}{ M étodo de estimación } & \\
\hline & \multicolumn{2}{|c|}{ M Rg } & \multicolumn{2}{|c|}{$M R z$} & \multicolumn{2}{|c|}{ M 3p } \\
\hline & CLF & PP & CLF & $P P$ & CLF & $P P$ \\
\hline A proximación normal & 1 & 0.30 & 3 & 0.90 & 2 & 0.60 \\
\hline EM \% promedio & 2 & 0.30 & 1 & 0.15 & 3 & 0.45 \\
\hline $\mathrm{EM} \%>10$ & 2 & 0.50 & 1 & 0.25 & 3 & 0.75 \\
\hline f & 2 & 0.30 & 3 & 0.45 & 1 & 0.15 \\
\hline Costos & 1 & 0.15 & 2 & 0.30 & $3^{1}$ & 0.45 \\
\hline TOTAL & & 1.55 & & 2.05 & & 2.40 \\
\hline
\end{tabular}

${ }^{1}$ Calificación asignada por requerimiento de dos ingresos al bosque

Factores de ponderación

A proximación normal $\quad 0.30$

EM \% > $10 \quad 0.25$

EM \% promedio $\quad 0.15$

f 0.15

Costos $\quad 0.15$

$C L F=$ calificación

$\mathrm{PP}=$ puntaje ponderado

Enseguida, la comparación entre la técnica muestral más eficiente $\mathbf{M R g}$ y el método tradicional MTr puso en evidencia la mayor exactitud de la primera en términos del error absoluto de estimación del volumen por parcela circular (figura 3) Este antecedente y las cifras inferiores de fracción muestral y de costos promedios de $\mathbf{M R g}$ determinaron en definitiva su calificación como método de mayor eficiencia para estimar el volumen por unidad secundaria.

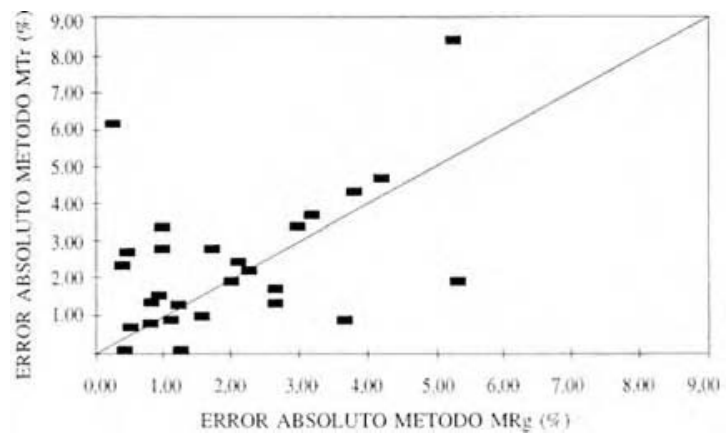

Figura 3. Error absoluto de estimación del método $\mathrm{MTr}$ comparado con el método $\mathrm{MRg}$.

Absolute estimation error of the MTi method compared with the MRg method.
Practicando el método seleccionado a nivel de unidad secundaria, la estimación de la media y total poblacionales del volumen bruto, mediante los estimadores del muestreo bietápico, se asoció a un error muestral de $182 \%$, es decn, superior en 82 unidades al máximo planificado (cuadro 6) El excesivo error muestral se atribuye al aporte significativo de la varianza entre unidades primarias a la varianza total, a raíz de la elevada heterogeneidad del volumen medio por rodal.

Los mismos parámetros poblacionales calculados en base a las estimaciones del VT/UMS de los cuatro métodos sometidos a estudio comprobaron que la eficiencia de éstos dependió fundamentalmente de los costos de operación de terreno, puesto que las diferencias en unidades de voIumen fueron menores al $\pm 05 \%$ (cuadro 7)

El aceptable grado de exactitud del muestreo dentro de las unidades secundarias se verificó mediante el residuo porcentual de 0 7\% observa do entre las estimaciones obtenidas a partir de los volúmenes reales por parcela y en base a muestreo mediante estimadores de regresion (cuadro 8). 


\section{CUADRO 6}

Parámetros poblacionales estimados mediante muestreo bietápico con probabilidad proporcional a la superficie de los rodales.

Estimated population parameters using two stage sampling with probability proportional to stand surface.

\begin{tabular}{|c|c|c|c|c|c|c|c|c|c|}
\hline \multirow{2}{*}{$\begin{array}{l}\text { Rodal } \\
\text { muestra }\end{array}$} & \multirow[b]{2}{*}{$\mathrm{Mi}$} & \multirow[b]{2}{*}{$\mathrm{mi}$} & \multicolumn{4}{|c|}{$\mathrm{m}^{3} / \mathrm{UMS}$} & \multirow[b]{2}{*}{ y̆i } & \multicolumn{2}{|c|}{ Varianza } \\
\hline & & & 1 & 2 & 3 & 4 & & VI & $\mathrm{V} 2$ \\
\hline 01 & 8.2 & 1 & 1.78 & & & & 1.78 & 31 & 0 \\
\hline 02 & 22.9 & 2 & 2.06 & 0.99 & & & 1.53 & 3.044 & 8.596 \\
\hline 03 & 34.3 & 1 & 2.58 & & & & 2.58 & 27.525 & 0 \\
\hline 04 & 43.6 & 1 & 1.87 & & & & 1.87 & 107 & 0 \\
\hline 09 & 10.8 & 1 & 1.17 & & & & 1.17 & 6.884 & 0 \\
\hline 12 & 111.5 & 3 & 046 & 1.60 & 1.36 & & 1.14 & 76.429 & 18.878 \\
\hline 13 & 120.2 & 2 & 1.66 & 1.83 & & & 1.74 & 1.353 & 1.214 \\
\hline 14 & 132.0 & 3 & 1.31 & 1.85 & 2.19 & & 1.78 & 452 & 12.144 \\
\hline 15 & 148.9 & 1 & 2.42 & & & & 2.42 & 74.251 & 0 \\
\hline 16 & 207.9 & 4 & 1.03 & 1.51 & 1.96 & 1.15 & 1.41 & 53.125 & 12.702 \\
\hline 17 & 48.8 & 2 & 2.51 & 3.04 & & & 2.78 & 62.540 & 4.717 \\
\hline 19 & 190.8 & 2 & 1.83 & 2.03 & & & 1.93 & 2.788 & 2.823 \\
\hline 24 & 168.5 & 4 & 0.55 & 0.16 & 1.29 & 1.25 & 0.81 & 252.564 & 17.973 \\
\hline 25 & 39.4 & 2 & 2.37 & 3.03 & & & 2.70 & 42.702 & 5.856 \\
\hline TOTAL & - & 29 & - & & - & - & - & 603.794 & 84.903 \\
\hline$\%$ & - & - & - & - & - & - & - & 87.7 & 12.3 \\
\hline
\end{tabular}

Media poblacional

Total poblacional

Varianza de la media

Varianza total poblacional

Error muestral

$\quad 45.8 \mathrm{~m}_{3}^{3} / \mathrm{ha}$
$2.633 .9 \mathrm{~m}^{3}$
149
49381.0
$18.2 \%$

$18.2 \%$

\section{CUADRO 7}

Parámetros poblacionales relativos a distintos métodos de estimación del volumen por parcela. Population parameters in relation to the different methods to estimate plot volume.

\begin{tabular}{|c|c|c|c|c|}
\hline \multirow{2}{*}{$\begin{array}{l}\text { Parámetro } \\
\text { poblacional }\end{array}$} & \multicolumn{4}{|c|}{ Método de estimación } \\
\hline & MRg & $\mathrm{MRz}$ & M3p & $\operatorname{MTr}$ \\
\hline Volumen medio $\mathrm{m}^{3} / \mathrm{ha}$ & 45.8 & 46.0 & 45.9 & 45.6 \\
\hline Volumen total $\mathrm{m}^{3}$ & 2.633 .9 & 2.643 .9 & 2.637 .0 & 2.619 .6 \\
\hline Diferencia con MRg \% & 00.0 & 0.4 & 0.2 & -0.4 \\
\hline EM \% & 18.2 & 18.5 & 18.3 & 18.0 \\
\hline Diferencia con MRg \% & 00.0 & 1.6 & 0.6 & -1.1 \\
\hline
\end{tabular}




\section{CUADRO 8}

Parámetros poblacionales calculados con VT/parc real y estimado mediante muestreo con estimadores de regresión.

Population parameters calculated with real and estimated VT/plot using samples with estimated regressions.

\begin{tabular}{|lrrr|}
\hline \multirow{2}{*}{$\begin{array}{l}\text { Parámetro } \\
\text { poblacional }\end{array}$} & & \multicolumn{2}{c|}{ Volumen/Parcela } \\
\cline { 3 - 4 } & & VTR & VTE - MRg \\
\hline Volumen medio & $\mathrm{m}^{3} / \mathrm{ha}$ & 37.7 & 37.4 \\
Volumen total & $\mathrm{m}^{3}$ & 2.164 .9 & 2.150 .3 \\
Diferencia con VTR & $\%$ & 00.0 & -0.7 \\
EM & $\%$ & 18.6 & 18.9 \\
Diferencia con VTR & $\%$ & 00.0 & 1.6 \\
\hline
\end{tabular}

VTR $\quad=$ volumen real por parcela

VTE MRg = volumen por parcela estimado mediante estimadores de regresión

Los estimadores del submuestreo con probabilidad variable proporcionaron además la estimación de las variables que se presentan en el cuadro 9.

\section{CUADRO 9}

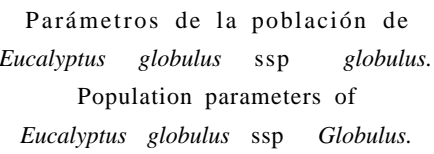

\begin{tabular}{|c|c|}
\hline Parámetro & Valor estimado \\
\hline 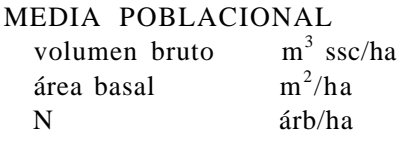 & $\begin{array}{c}45.81 \\
9.0475 \\
590\end{array}$ \\
\hline $\begin{array}{l}\text { TOTAL POBLACIONAL } \\
\begin{array}{l}\text { volumen bruto } \\
\mathrm{N}\end{array} \\
\begin{array}{l}\mathrm{m}^{3} \mathrm{ssc} \\
\mathrm{arb}\end{array}\end{array}$ & $\begin{array}{l}2633.9 \\
33933\end{array}$ \\
\hline $\begin{array}{l}\text { ERROR MUESTRAL }{ }^{1} \\
\text { volumen bruto } \\
\% \\
\text { área basal } \\
\mathrm{N}\end{array}$ & $\begin{array}{l}18.2 \\
15.9 \\
18.9\end{array}$ \\
\hline $\begin{array}{l}\text { LIMITES CONFIDENCIALES } \\
\begin{array}{l}\text { volumen bruto } \\
\text { área basal }\end{array} \\
\begin{array}{l}\mathrm{m}^{2} / \mathrm{ha} \\
\mathrm{N}\end{array} \\
\text { aib/ha }\end{array}$ & $\begin{array}{cl}37.47 & -54.15 \\
7.6089 & -10.4860 \\
479 & 702\end{array}$ \\
\hline
\end{tabular}

$\mathrm{dg}=13.97 \mathrm{~cm}$

$\mathrm{h}=13.93 \mathrm{~m}$

$1 \quad$ Calculado con "t" $(\mathrm{gl}=13 ; \mathrm{p}=0.05)=2.1604$

\section{CONCLUSIONES}

El muestreo bietápico con probabilidad variable se ajusta aceptablemente al plan de inventario de una población de rodales de un amplio rango de superficies, característica frecuente en plantaciones de forestería participativa Sin embargo, el diseño muestral demuestra limitaciones para controlar el error muestral máximo planificado debido a su sensibilidad ante la vananza entre unidades primarias.

A nivel de unidades secundarias, el muestreo con estimadores de regresión se constituyó en la técnica más eficiente para estimar el volumen bruto por parcela VT/UMS El grado de exactitud y la reducción de costos alcanzado por este método justificaron, además, la aplicación de técnicas muéstrales para la estimación del VT/UMS.

A diferencia del método tradicional MTr, el muestreo con estimadores de regresión manifiesta también la cualidad de definir la fracción de árboles que deben construir la muestra y el error máximo de las estimaciones.

No obstante, con el propósito de estimar existencias netas, la metodología de la técnica MRg debe complementarse aplicando, por ejemplo, factores de transformación (cull factors).

\section{REFERENCIAS}

BENITEZ S., P. 1992. Aplicación del muestreo con probabilidad proporcional a la predicción 3p a poblaciones pequeñas. Tesis de grado. Valdivia, Chile, $43 \mathrm{p}$

BONNOR G., M. 1972. "Cost of a small forest inventory". Canadian Journal of Forest Research. 2:45-48.

COCHRAN, W. 1978. Técnicas de muestreo. Trad. por Eduardo Casas. México D.F., Continental S.A. 507 p.

DE VRIES, P. 1986. Sampling theory for forest inventory: A teach-yourself course. New York, EE.UU., Springer, p. 88103.

GROSENBAUGH, L.R. 1976. "Approximate sampling variance of adjunted 3p sampling estimate". Forest Science 22: 173178.

LOETSCH, F., F. ZOHRER, K.E. HALLER. 1973. Forest inventory. München, Germany, BLV Verlagsgesellchaft, V.2: $380-401$

PELZ, D.R. 1980. Stichproben in kleinflächigen Beständen. Forstw. Cbl. 99: 55-60

SCHEAFFER, R., W. MENDENHALL, L. OTT. 1986 Elementary survey sampling. $3^{\text {th }}$ ed., Boston, EE.UU., PWS Publishers. 278 p. 